\title{
Preflight calibration and testing of EUSO-SPB in the lab and the desert
}

\author{
J. Eser ${ }^{* 1 \dagger}$, J. Adams², S. Bacholle ${ }^{1}$, A. Cummings ${ }^{1}$, A. Diaz Damian ${ }^{3}$, E. Kuznetsov², \\ M. Mustafa ${ }^{2}$, W. Painter ${ }^{4}$, L. Piotrowski ${ }^{5}$, L. Wiencke ${ }^{1}$ \\ ${ }^{1}$ Colorado School of Mines \\ ${ }^{2}$ University of Alabama, Huntsville \\ ${ }^{3}$ IRAP \\ ${ }^{4}$ Karlsruhe Institute of Technology \\ ${ }^{5}$ RIKEN \\ for the JEM-EUSO Collaboration
}

The Extreme Universe Space Observatory on a super pressure balloon (EUSO-SPB) instrument is a pathfinder for space based cosmic ray fluorescence detectors. It is in preparation to be launched from Wanaka, New Zealand by NASA with a target launch date of 25th of March 2017. The optical system of the instrument consists of 2 Fresnel lenses. The focal surface is a camera with 2304 pixel and a time resolution of $2.5 \mu$ s build up with a modular design. The lenses and the camera where separately characterized in the laboratory. Before using the instrument for a full scale test in the field, a flat fielding was performed in the lab as well. The field tests were performed in the west Utah desert at the Telescope Array (TA) site in September 2016. The test included star observation and background measurements. An absolute calibration with an $365 \mathrm{~nm}$ UV-LED and with a laser "test beam" system. Laser tracks were measured at a distance of $24 \mathrm{~km}$ in various geometries to obtain a full characterization of the detector capability to measure cosmic rays. We will describe briefly the test system and the performed test and present the results of the laboratory and field tests of EUSO-SPB.

35th International Cosmic Ray Conference - ICRC2017-

10-20 July, 2017

Bexco, Busan, Korea

${ }^{*}$ Speaker.

†jeser@mines.edu 


\section{Introduction and Motivation}

The Extreme Universe Space Observatory on a Super Pressure Balloon (EUSO-SPB) instrument is a prototype for space based cosmic ray fluorescence detectors. EUSO-SPB was launched by NASA from Wanaka, NZ on the 25th of April 2017. The instrument flew for 12 days and $4.5 \mathrm{~h}$ before it was terminated early over the Pacific ocean. A full description of the mission is given in [1]. A detailed description of the detector can be found in [2]. Briefly the detector focuses lights with 2 Fresnel lenses onto the camera. The camera is photomultiplier based with 2304 pixel and capable of single photoelectron (pe) counting. It is sensitive in the UV range and has a time resolution of $2.5 \mu \mathrm{s}$. A trigger [3] gives the potential of detecting the first cosmic ray via fluorescence from sub orbital space.

The preflight calibration can be separated in two major parts, the laboratory measurements for single components (Photo Detection Module, lenses) and the field tests for an end-to-end characterization. The field test were necessary to achieve a full scale test of the instrument and estimate its capability of measuring tracks from an extensive air shower (EAS). In addition a flat fielding was performed in Wanaka.

\section{Laboratory Tests}

\subsection{PDM Calibration}

The relative and absolute photometric calibration of the Photo Detection Module (PDM) were performed at APC in Paris. The photometric calibration provides a relationship between the amount of photons arriving at the detector and the measured signal.

For the relative calibration 36 independent measurements, one for each Multianode Photomultipliere Tube (MAPMT) were combined. For each measurement the port of an integrating sphere was pointed to the center of one of the 36 MAPMTs from a distance of $49 \mathrm{~cm}$. A DC-UV-LED with the wavelength $378 \mathrm{~nm}$ served as light source. A uniform illumination of the PDM above 99.87\% could be achieved. Out of the combined measurement the relative efficiency of each pixel normalized to the mean of the full PDM was calculated.

For the absolute calibration the PDM was uniform illuminated with the same UV-LED (see figure 1a). The light flux at the PDM position was measured by a photodiode that had a NIST traceable absolute calibration (NIST PD) [6]. In the next step the relative measurement were weighted by the measured intensity at the PDM position normalized to a pixel size. The result of these measurement is displayed in figure $1 \mathrm{~b}$.

The uncertainties of this measurement are dominated by the uncertainty of the positioning of the NIST at the PDM position as well as the uncertainty of the active area of the NIST. In average for all pixel 1 photon produces $0.305 \pm 0.030$ pe.

\subsection{Fresnel lens characterization}

The Fresnel lens systems were tested in a $1 \mathrm{~m}$ diameter, parallel beam of UV light in a test stand at the Colorado School of Mines (figure 2). The calibration source was a fiber coupled DC-UV-LED. The fiber bundle was placed in the focal point of a diffraction limited $1 \mathrm{~m}$ mirror. The fiber was needed to minimize the obstruction of the reflected light, more important it allowed 


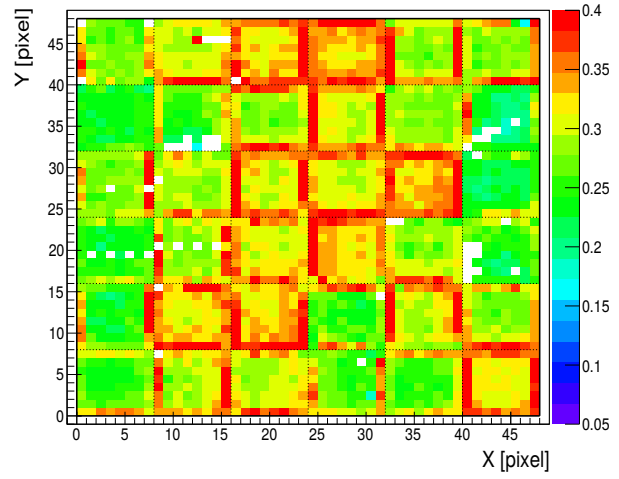

(a) Example frame of the uniformly illuminated PDM at the standard setting; color code is calibration factor

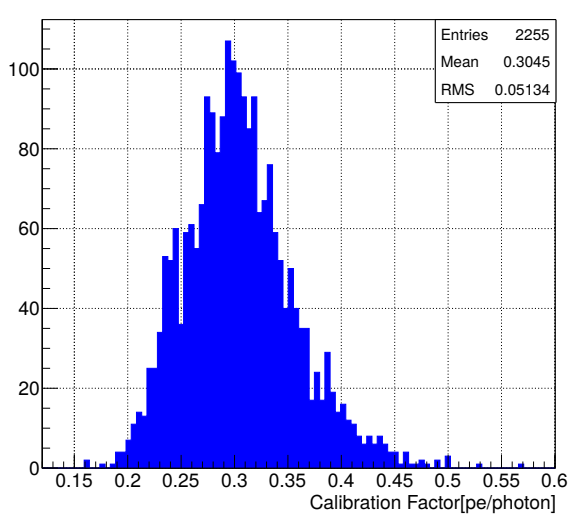

(b) Calibration factor for each pixel

Figure 1: Absolute calibration of the PDM in the laboratory, APC, 2016 with a 375 nm-LED

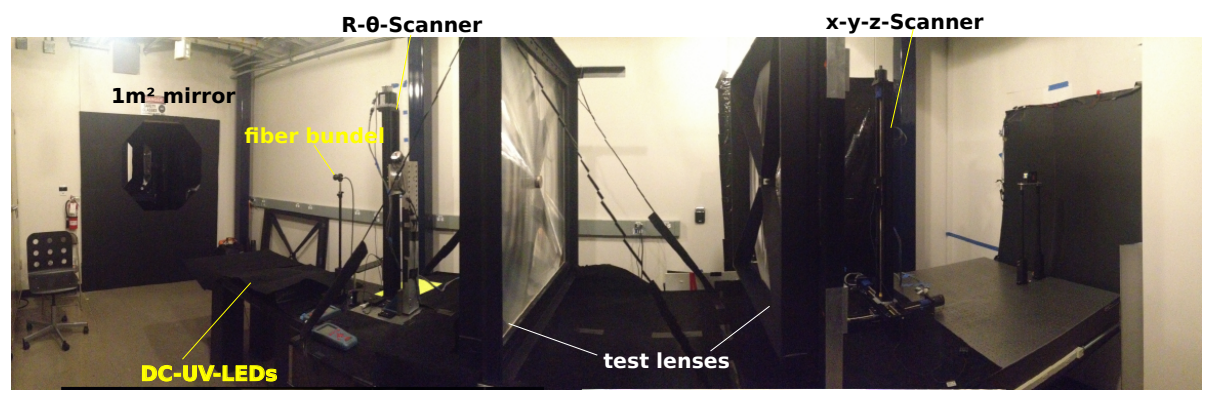

Figure 2: EUSO-SPB lens test setup

to exchange the LEDs without touching the alignment of the collimator. $10 \mathrm{~nm}$ bandpass filters were used to select a narrow wavelength window around the target wavelengths 340, 355, 370 and $390 \mathrm{~nm}$.

NIST-photodiodes [6] measured the light flux entering the lens system and the flux in the lens focal point. An R- $\theta$-"windmill"-scanner sampled the incoming light flux upstream of the lenses at 408 points. The total flux was calculated by integrating over these points. The lens system underwent three different tests: a scan along the optical axis, a cube scan around the rough predefined focal point and a slit scan perpendicular to the optical axis. In each test all 4 wavelength were used. The "cube scan" was done by taking measurements forming a 3D grid which contains images of the Point Spread Function (PSF) at different intervals along the optical axis (figure 3a). The area in which the signal is higher than $10 \%$ of the maximum signal is defined as the PSF. The throughput of the lenses, mean the percentage of light focused in the focal point compared to the light send into the lens system, was obtained by calculating the encircled energy of the PSF for each image plane. This method gives us the throughput value of the system at a given radius from the centroid of the PSF which allows us to determine the throughput of the system for each wavelength at different distances along the optical axis. See figure 3b. A lens system containing three lenses was also tested. The 3rd (middle) diffraction lens, a prototype, corrected for chromatic aberration 


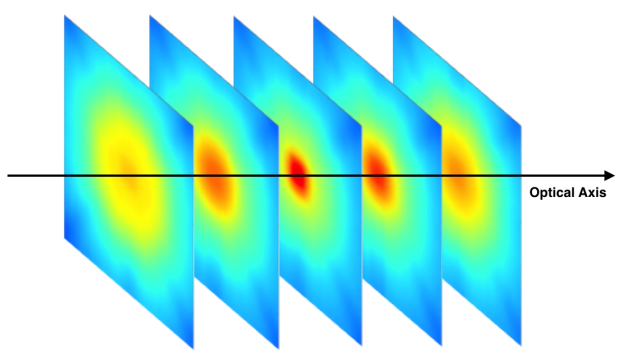

(a)

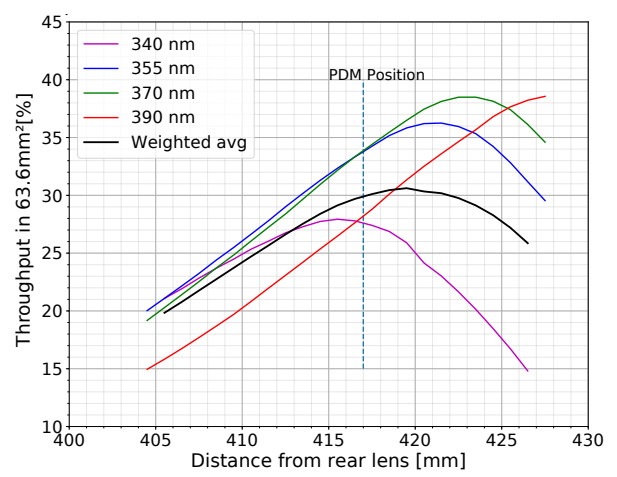

(b)

Figure 3: Result of the lens tests. a) visulalisation of cube scan, b) throughput in a 3 by 3 pixel grid

Table 1: Lens test results for the 2 lens system (flight configuration) and the 3 lens system (containing diffractive lens)

\begin{tabular}{l|c|c} 
& 2 lens system & 3 lens system \\
\hline Throughput & $30 \%$ & $12 \%$ \\
Spot size & $3 \mathrm{px} \times 3 \mathrm{px}$ & $1 \mathrm{px}$ \\
Distance between focal points & $15 \mathrm{~mm}$ & $1 \mathrm{~mm}$ \\
340 and $390 \mathrm{~nm}$ & &
\end{tabular}

and minimized the PSF. Even though the diffractive lens reduced the aberration and the PSF as expected, the loss in throughput was too large (2-2.5 times smaller as the 2 lens system). Table 1 contains a summary of the tests.

\subsection{Flat Fielding}

A flat fielding map is to correct for non uniform sensitivity over the focal surface including possible distortions in the optical path. To flat field this detector a Tyvek1056 covered screen was located $4.65 \mathrm{~m}$ in front of the detector in a darkened room. The screen was illuminated by a $365 \mathrm{~nm}$ LED pulse The reflected light from the screen was assumed to be totally diffused. The LED was pulsed at several different intensity levels to cover the dynamic range of the detector. The counts in each pixel were normalized to the average count over the full camera. The flat fielding was repeated in Wanaka, NZ prior to the launch in flight configuration with the instrument hanging from a crane $5 \mathrm{~m}$ above a Tyvek1025D screen on the ground which allowed to spin the instrument in front of the screen. To illustrate the effect of the flat fielding figure $4 \mathrm{a}$ displays a sky frame during the flight, figure $4 \mathrm{~b}$ shows the same sky frame with applied flat frame.

\section{Field Tests}

\subsection{The Campaign and the Laser System}

For an end-to-end test of the EUSO-SPB instrument in the field the fully assembled detector 


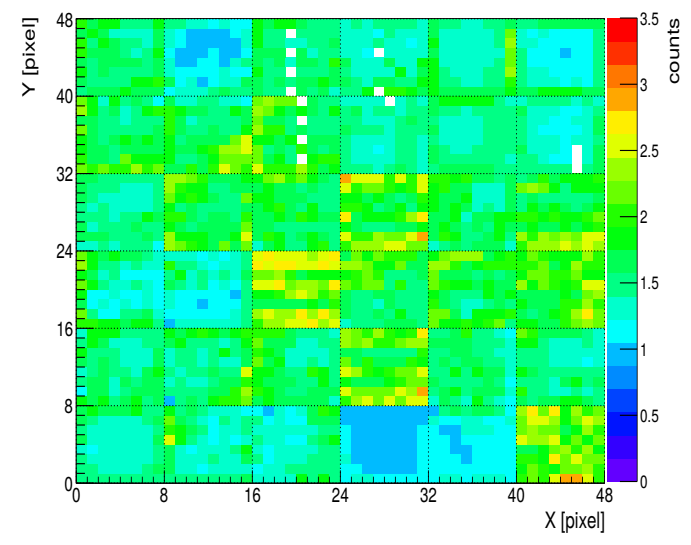

(a)

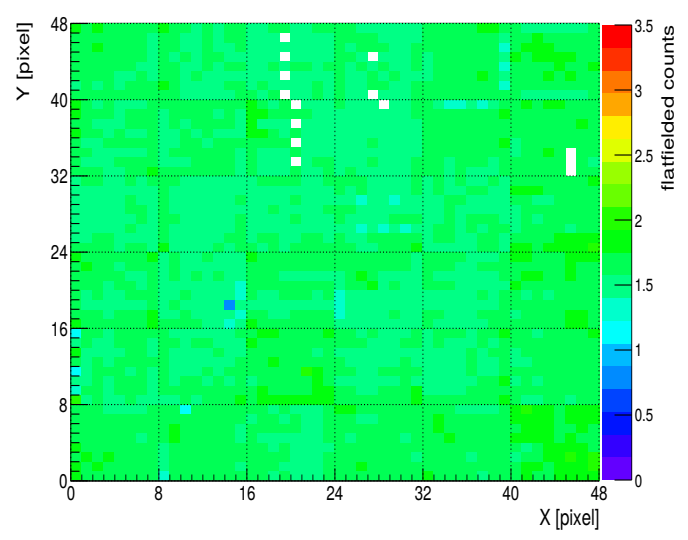

(b)

Figure 4: a) Flight night sky without flat frame, b) Flight night sky with flat frame obtained in Wanaka

was transported $845 \mathrm{~km}$ from Golden, Colorado to the Telescope Array (TA) site in Delta, Utah and back. This transport presented significant logistical challenges. A custom trailer and dolly were designed and used to minimize vibration during the transportation. Prior to the transport of the instrument a dummy load was driven and the acceleration was measured. An additional safety measure was the use of a pilot car in front to warn the instrument driver behind of potential road hazards. Accelerometers were installed to measure the vibration during the transport. The instrument experienced less than $1 \mathrm{~g}$ impacts during the trip. EUSO-SPB was set up next to another JEM-EUSO prototype system, EUSO-TA [4], on Black Rock Mesa, one of the fluorescent detector site of the TA. Four hours after arrival first tracks of the TA central laser facility were observed.

For the 8 day campaign, the telescope was tilted up by $7.8^{\circ}$ and recorded hundreds of thousands of laser shots in different directions and at different energies. The Global Light System (GLS) prototype [5] was used. This laser system is portable and the $355 \mathrm{~nm}$ beam can be steered in any direction with a $0.2^{\circ}$ precision. The energy range is between $250 \mu \mathrm{J}$ and $80 \mathrm{~mJ}$. The beam is randomly polarized within $4 \%$ and the energy is monitored with an accuracy of 5\%. The system was tested in multiple earlier campaigns. The GLS site and the energy sweep geometry are shown in figure 5 .

An absolute calibration was performed in addition to a measurement of the energy threshold. The recorded data contains, in addition to the laser and calibration events, background data including airplanes, stars and meteors.

\subsection{Absolute Calibration with LED}

One goal of the field test was to perform an end-to-end photometric calibration of the detector. At a distance of $45 \mathrm{~m}$, a $365 \mathrm{~nm}$-LED was mounted on a portable antenna mast. This LED was temperature stabilized to guarantee a well characterized calibration source.

Calibration data was taken for 9 different LED settings. The LED was operated in pulsed mode. Each pulse had a duration of $50 \mu$ s (equal to 20 GTUs). In the analysis only the 15 center GTUs are used to avoid rising and falling edge effects of the LED. The calibration constant is obtained 


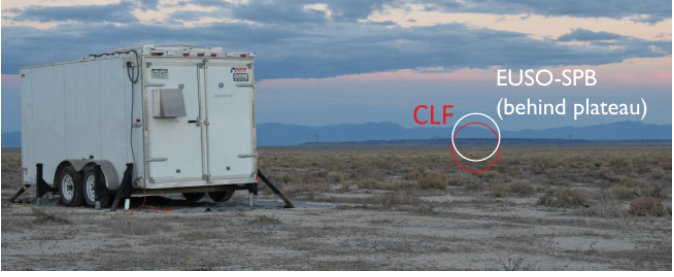

(a) roving laser GLS prototype system

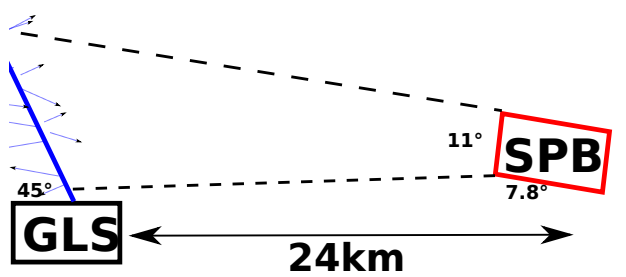

(b) Geometry sketch of laser beam used to estimate trigger efficiency

Figure 5: The GLS prototype system and it use during the field tests

by comparing the measured signal in photoelectron counts after applying the flat fielding and subtracting the background noise with the estimated number of photons arriving at the aperture. This number was calculated using the measured distance from the front lens to the LED and the absolute calibration of the LED's luminosity. An example LED event is shown in figure 6a. The plot $6 \mathrm{~b}$ shows the distribution of the photon to photoelectron conversion factor using different voltages and positions.

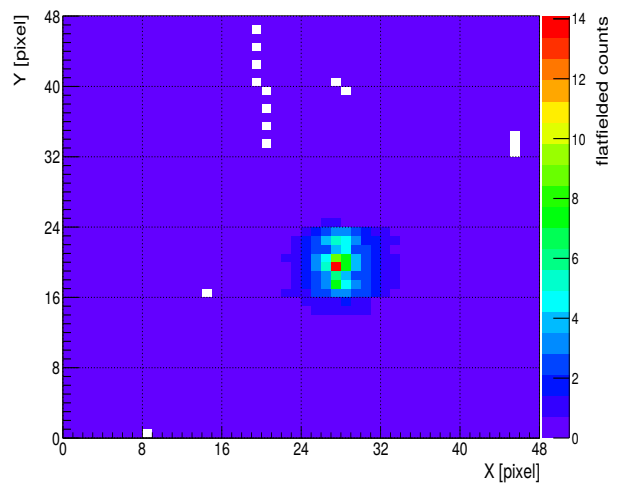

(a) Example LED pulse measured by SPB

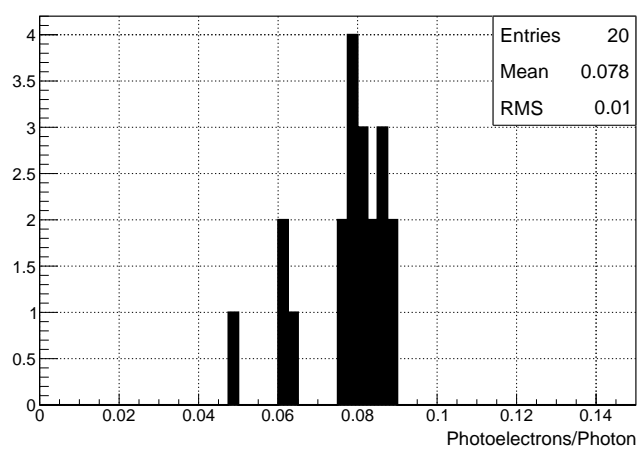

(b) Distribution of the absolute calibration factor for all positions and LED voltages

Figure 6: Photometric absolute calibration of EUSO-SPB as measured during the Utah field campaign in 2016

The photometric calibration factor of EUSO-SPB is estimated to $0.078 \pm 0.010 \mathrm{pe} /$ photon. This agrees with the lab measurements.

\subsection{Estimation of Trigger Efficiency and Energy Threshold}

To estimate the trigger efficiency (energy threshold) the GLS-prototype laser system was used to create shower like signals in the detector with a well characterized energy and direction. For the energy sweep test, the laser was tilted $45^{\circ}$ away from EUSO-SPB in the detector plane, in a distance of $24 \mathrm{~km}$. Combined with the $7.8^{\circ}$ tilt of EUSO-SPB, this would be the equivalent of a cosmic ray with an inclination angle of 53 degrees (see figure 7a). The energy of the laser was changed between $4 \mathrm{~mJ}$ and $500 \mu \mathrm{J}$ in fine steps. 100 shots were fired at each energy setting. To obtain the true trigger rate for each energy setting, noise trigger were removed. Also the effect of 
clouds on trigger enhancement or reduction was consider by analyzing the light profile shapes as well as long exposure pictures of the sky. The resulting trigger rate compared to laser energy is plotted in figure $7 \mathrm{~b}$.

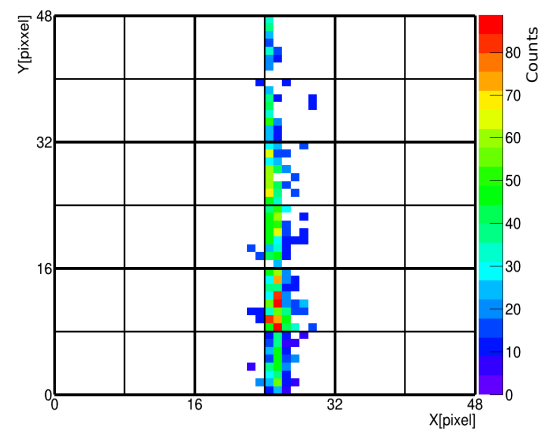

(a) example laser track

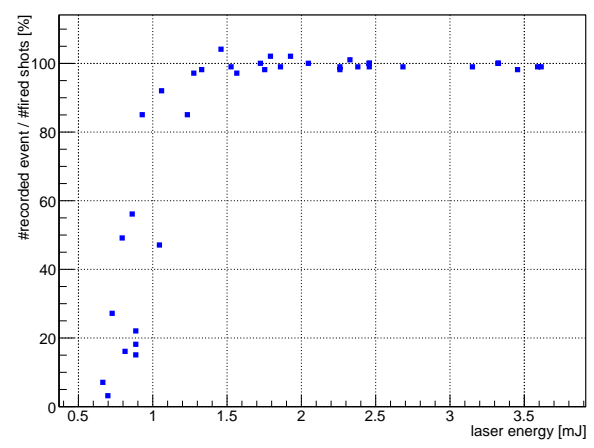

(b) Trigger efficiency for given laser energy

Figure 7: Trigger efficiency of EUSO-SPB as measured during the Utah field campaign in 2016

To convert laser to cosmic ray energy simulations were used. The amount of photons produced by an extensive air shower arriving at the detector's aperture was simulated. Typical geometry proton primaries were simulated for EUSO-SPB in flight configuration with energies between $1 \mathrm{EeV}$ to $30 \mathrm{EeV}$. The laser was simulated with the geometry utilized in field testing. The laser profile was scaled to match the maximum number of photons per pixel per GTU between the two processes. In addition the different atmospheric and geometry effects during the flight compared to the ground were taking into account. This scaling factor was then converted to find the equivalent laser energy of an EAS as seen from the balloon. More details about the trigger efficiency estimation can be found in [7]. The energy threshold (equals trigger efficiency of 50\%) of EUSO-SPB was determined to be $3 \mathrm{EeV}$.

This test was also performed with the three lens setup of EUSO-SPB, to evaluate the performance in a realistic environment. The results confirmed the laboratory measurements (described in 2.2) and resulted in an trigger threshold of about two times higher.

\subsection{Field of View Measurements}

The FoV was checked using laser sweeps and crossing stars. An astronomy standard method to estimate a telescopes field of view uses the change of inclination of a identified bright star within the view over time. An example star observation is shown in figure 8 . This was done for five bright stars and resulted in a FoV estimation of $11.10 \pm 0.15^{\circ}$. The biggest uncertainty for this method is the uncertainty in the star position.

A cross check was made by sweeping the laser in $2^{\circ}$ steps across the perpendicular plane to the laser-detector-plane (figure 9). The pointing direction of the laser is known better than $0.2^{\circ}$. The position of the GLS prototype is known within GPS uncertainties. The result of this method is a FoV of $11.2 \pm 0.1^{\circ}$. The two methods are consistent within errors. The average yields a pixel FoV of $0.23^{\circ}$. 


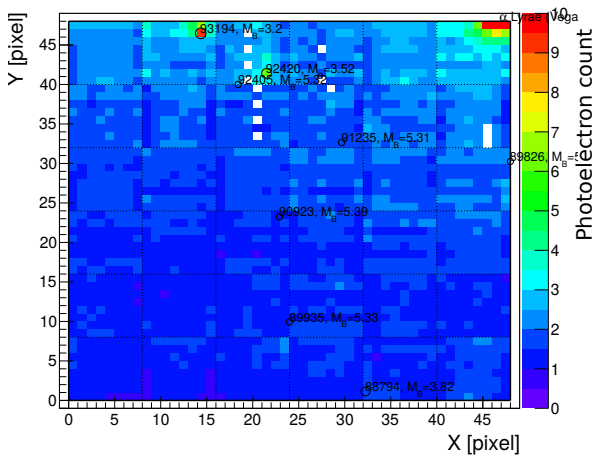

Figure 8: Night picture of Utah sky with stars marked used for FoV calculation

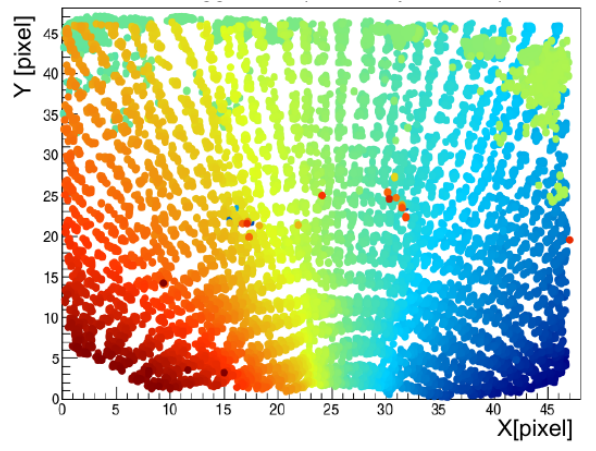

Figure 9: Trigger plot for laser sweep (and some background events); blue: early, red: late

\section{Summary}

EUSO-SPB was calibrated and characterized before the launch in April 2017. Single components were tested in a laboratory environment. These tests lead to a calibration factor of $0.30 \pm 0.03 \mathrm{pe} /$ photon. The lens test showed a PSF of $10 \mathrm{~mm}$ with a throughput of $30 \%$. During the field test it was possible to perform a photometric calibration of the instrument. The result conversion is $0.08 \pm 0.01 \mathrm{pe} /$ photon. The FoV measurement estimated a FoV of $11.1 \pm 0.2^{\circ}$. Using laser tracks the energy threshold for detecting cosmic rays was found to be $3 \mathrm{EeV}$.

Acknowledgment: This work was partially supported by NASA grants NNX13AH55G, NNX13AH53G, the French Space Agency (CNES), the Italian Space Agency through the ASI INFN agreement n. 2017-8-H.0, the Basic Science Interdisciplinary Research Projects of RIKEN and JSPS KAKENHI Grant (22340063, 23340081, and 24244042), the Deutsches Zentrum für Luft und Raumfahrt, and the 'Helmholtz Alliance for Astroparticle Physics HAP' funded by the Initiative and Networking Fund of the Helmholtz Association (Germany). We also acknowledge the Telescope Array Collaboration for the use of facilities in Utah as well as the Goddard Space Flight Center for the loan of the mirror used for the lens test.

\section{References}

[1] L. Wiencke et al. (JEM-EUSO Collaboration), EUSO-SPB Mission and Science in Proc. 35th ICRC, (Busan), (2017).

[2] S. Bacholle et al. (JEM-EUSO Collaboration), The EUSO-SPB Instrument in Proc. 35th ICRC, (Busan), (2017).

[3] J. Bayer et al. (JEM-EUSO Collaboration), The trigger logic of EUSO-SPB and its performance in Proc. 35th ICRC, (Busan), (2017).

[4] J.H. Adams et al. (JEM-EUSO Collaboration), Ground-based tests of JEM-EUSO components at the Telescope Array site, "EUSO-TA", Experimental Astronomy Volume 40, Issue 1, pp 301-314, (2015).

[5] P. Hunt et al. (JEM-EUSO Collaboration), The JEM-EUSO global light system laser station prototype in Proc. 34th ICRC, (The Hauge), (2015).

[6] http://www.ophiropt.com/.

[7] A. Cummings, Field Testing of EUSO-SPB: Logistics and First Results, Master's thesis, Colorado School of Mines, 2017. 